\title{
Facile synthesis of silver/silver thiocyanate (Ag@AgSCN) plasmonic nanostructures with enhanced photocatalytic performance
}

\author{
Xinfu Zhao ${ }^{1}$, Dairong Chen ${ }^{1,2}$, Abdul Qayum ${ }^{1}$, Bo Chen ${ }^{* 2}$ and Xiuling Jiao ${ }^{* 1,2}$
}

\author{
Full Research Paper \\ Address: \\ ${ }^{1}$ School of Chemistry and Chemical Engineering, Shandong \\ University, Jinan 250100, PR China and ${ }^{2}$ National Engineering \\ Research Center for Colloidal Materials, Shandong University, Jinan \\ 250100, PR China \\ Email: \\ Bo Chen* - sduchenbo@126.com; Xiuling Jiao* - jiaoxl@sdu.edu.cn \\ * Corresponding author \\ Keywords: \\ Ag@AgSCN; degradation of oxytetracycline; plasmonic photocatalyst; \\ stability
}

Open Access

Beilstein J. Nanotechnol. 2017, 8, 2781-2789.

doi:10.3762/bjnano.8.277

Received: 12 August 2017

Accepted: 08 November 2017

Published: 22 December 2017

This article is part of the Thematic Series "Energy conversion, storage and environmental remediation using nanomaterials".

Guest Editor: W.-J. Ong

(C) 2017 Zhao et al.; licensee Beilstein-Institut.

License and terms: see end of document.

\begin{abstract}
A nanostructured plasmonic photocatalyst, silver/silver thiocyanate $(\mathrm{Ag} @ \mathrm{AgSCN})$, has been prepared by a simple precipitation method followed by UV-light-induced reduction. The ratio of Ag to silver thiocyanate (AgSCN) can be controlled by simply adjusting the photo-induced reduction time. The formation mechanism of the product was investigated based on the timedependent experiments. Further experiments indicated that the prepared $\mathrm{Ag} @ \mathrm{AgSCN}$ nanostructures with an atomic ratio of $\mathrm{Ag} / \mathrm{AgSCN}=0.0463$ exhibited high photocatalytic activity and long-term stability for the degradation of oxytetracycline (84\%) under visible-light irradiation. In addition to the microstructure and high specific surface area, the enhanced photocatalytic activity was mainly caused by the surface plasmon resonance of Ag nanoparticles, and the high stability of AgSCN resulted in the longterm stability of the photocatalyst product.
\end{abstract}

\section{Introduction}

In the past decade, water decontamination technology has attracted great attention due to the increasing health risk that water contamination poses to humankind. The removal of pollutants has been intensively investigated in recent years, and various methods such as adsorption, biodegradation, photocatalytic degradation and chemical oxidation have been developed [1-4]. Among them, photocatalytic degradation is considered as one of the most effective strategies due to its high removal effi-

ciency and environmental friendlessness. For example, as a typical semiconductor, $\mathrm{TiO}_{2}$ exhibits high photocatalytic degradation performance against a large number of organic pollutants [5-7]. However, it is difficult to obtain a high photocatalytic activity under visible-light irradiation with $\mathrm{TiO}_{2}$ as a catalyst due to its wide bandgap of $3.2 \mathrm{eV}$, which limits its practical application. Therefore, the development of new photocatalysts with visible-light catalytic performance, high surface active 
sites and long life of separated electron and hole pairs, has become a hot research topic in recent years.

Ag-based semiconductors are well known due to their excellent visible-light catalytic properties, but their easy inactivation limits their utilization in practice. Aimed at this problem, a series of methods, such as doping, surface sensitization, heterojunctions and noble-metal plasma, have been adopted to improve the visible-light catalytic performance of Ag-based materials [8-10]. Among them, plasmonic photocatalysts consisting of $\mathrm{Ag} / \mathrm{AgX}(\mathrm{X}=\mathrm{Cl}, \mathrm{Br}, \mathrm{I})$ have exhibited improvement in separation of photogenerated electrons and holes [1118]. However, these Ag-based photocatalysts, $\mathrm{Ag} / \mathrm{AgX}(\mathrm{X}=\mathrm{Cl}$, $\mathrm{Br}, \mathrm{I})$, suffer from instability during the recycling application, mainly due to the decomposition of $\mathrm{AgX}(\mathrm{X}=\mathrm{Cl}, \mathrm{Br}, \mathrm{I})$ under irradiation [19-22]. Therefore, the development of visible-light catalysts with both high catalytic activity and long-term stability is of great importance.

As one of the Ag-containing semiconductor materials, AgSCN exhibits superior stability under irradiation [23,24]. The relatively large bandgap of $\mathrm{AgSCN}$ (3.4 eV) makes it only ultraviolet light active, largely limiting the wide utilization of the solar light energy spectrum. The addition of Ag on AgSCN structures can not only improve the utilization of visible light, but can also trap the photogenerated electrons to enhance the catalytic efficiency and stability. In the present work, a nanostructured Ag@AgSCN plasmonic photocatalyst was achieved by using hydrazine hydrate as a reducing agent, followed by a UV-light-induced reduction, in which the ratio of Ag to AgSCN can be controlled by simply adjusting the photo-induced reduction time. The degradation of a representative contaminate, oxytetracycline, which often exists in contaminated water, was used to test the photocatalytic performance of Ag@AgSCN.
Compared with previous reports, the as-prepared product had regular morphology, more active sites and a higher specific surface area [23-28], which led to high photocatalytic activity. More importantly, the activity toward the degradation of oxytetracycline after the fifth cycle was almost the same as for the first cycle, which is notable for a Ag-based catalyst.

\section{Results and Discussion \\ Ag@AgSCN nanostructures}

Ag@AgSCN nanostructures were synthesized by a simple precipitation method, followed by UV-light-induced reduction. As shown in Figure 1a, all the XRD patterns of the $\mathrm{Ag} @ \mathrm{AgSCN}$ nanostructures formed at different irradiation times can be indexed to monoclinic AgSCN (JCPDS file, No. 72-1176). No obvious reflections of face-centered cubic Ag can be observed even with an irradiation time as long as $3 \mathrm{~h}$, mainly due to the small amount of Ag derived from the high stability of AgSCN. An SEM image of the sample $M_{2}$ is shown in Figure $1 \mathrm{~b}$ as a representation. It can be seen that the product is sphere-like nanostructures with uniform size of $\approx 2.0 \mu \mathrm{m}$. Further observation of the high-resolution SEM image (inset of Figure 1b) reveals that the nanostructures are composed of close-packed nanoplates. The large nanoplates with a thickness of tens of nanometers firstly close arrange into crisscross structures, and there are many small plates in the crossed gap to form the sphere-like nanostructures. The corresponding EDS analysis indicates that only elemental $\mathrm{Ag}, \mathrm{C}, \mathrm{N}$, and $\mathrm{S}$ are detected in the sample (Supporting Information File 1, Figure S1), showing the high chemical purity of the product. The selected area electron diffraction (SAED) pattern of a single particle (Figure 1c) displays a set of bright spots, obviously suggesting the high crystallinity of the product. Based on calculations, the spots can be indexed to diffraction from the $(\overline{2} 23)$ and (332) planes of the monoclinic AgSCN. The SAED pattern also reveals that
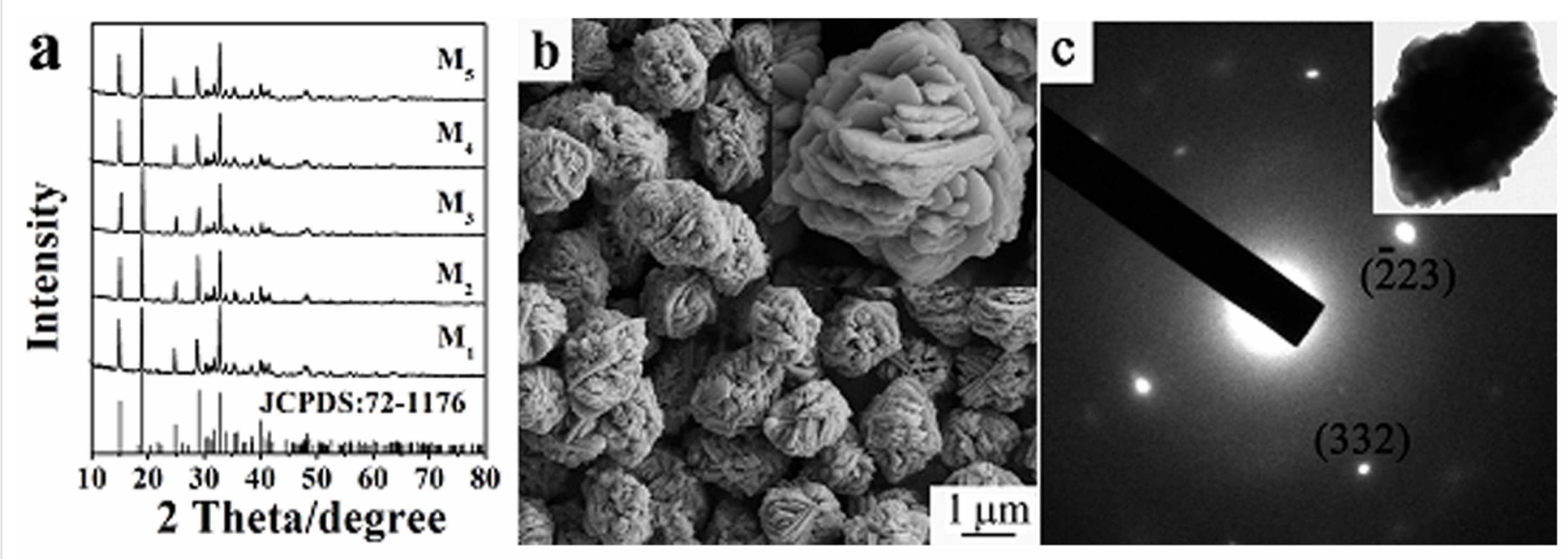

Figure 1: (a) XRD patterns of Ag@AgSCN nanostructures with different molar ratios of Ag to AgSCN. An (b) SEM image and (c) SAED pattern of the Ag@AgSCN nanostructure (sample $\mathrm{M}_{2}$ ). 
the oriented attachment of the nanoplates composed the $\mathrm{Ag} @ \mathrm{AgSCN}$ nanostructures.

To determine the Ag content in the Ag@AgSCN nanostructures, elemental analysis was conducted, and the results are shown in Table 1. Before UV irradiation, the molar ratio of Ag to $\mathrm{AgSCN}$ in the product is 0.024 . With the extension of the UV irradiation time, the content of $\mathrm{Ag}$ in the product gradually increases, and the molar ratio of $\mathrm{Ag}$ to $\mathrm{AgSCN}$ reaches 0.0775 after being irradiated for $3 \mathrm{~h}$, indicating the slow reduction of AgSCN under UV irradiation.

UV-vis diffuse reflectance spectra of $\mathrm{M}_{0}, \mathrm{M}_{1}, \mathrm{M}_{2}, \mathrm{M}_{3}, \mathrm{M}_{4}$, and $\mathrm{M}_{5}$ are shown in Figure 2a. Here, the characteristic absorption of AgSCN appears at 200-350 $\mathrm{nm}$ and that from the surface plasmon resonance of $\mathrm{Ag}$ particles is above $350 \mathrm{~nm}$. The absorption peak of silver nanoparticles becomes gradually stronger as the content of $\mathrm{Ag}$ increases, indicating that the sunlight utilization efficiency increases steadily. During UV irradiation, many defects are formed in $\mathrm{AgSCN}$, including differ- ent interstitial sites and vacancies [29]. The presence of silver particles not only improves the photocatalytic efficiency, but also enhances the electric field strength around AgSCN due to the surface plasmon resonance, which in turn enhances the optical transition of midgap defect states of AgSCN. All these conditions contribute to the strong absorption of $\mathrm{Ag} @ \mathrm{AgSCN}$ in both the UV and visible region, which is beneficial for application as a visible-light catalyst. The bandgap of AgSCN can be determined according to the Kubelka-Munk equation, which is estimated as $3.4 \mathrm{eV}$ for sample $\mathrm{M}_{0}$ (Figure $2 \mathrm{~b}$ ), and the valence band value of $1.12 \mathrm{eV}$ is obtained from [30]. Therefore, the conduction band position can be determined to be $-2.28 \mathrm{eV}$. The bandgap of sample $\mathrm{M}_{2}$ is estimated as $3.2 \mathrm{eV}$, where the change in the value of the bandgap of $\mathrm{AgSCN}$ may be due to the surface plasmon resonance of $\mathrm{Ag}$ nanoparticles under visible light. In this process, electrons in the metal from the Fermi level directly transfer to the conduction band of $\mathrm{AgSCN}$, and the vacancies remain on the surface of the Ag particles. A dipole-based resonance energy can directly excite semiconductors to produce photogenerated electron-hole pairs

\begin{tabular}{|c|c|c|c|c|c|}
\hline Sample & $\begin{array}{l}\text { Weight of Ag@AgSCN } \\
(\mathrm{mg})\end{array}$ & $\begin{array}{l}\text { Atomic content of S } \\
(\%)\end{array}$ & $\operatorname{AgSCN}(\mathrm{mol})^{\mathrm{a}} \times 10^{-5}$ & $\mathrm{Ag}(\mathrm{mol})^{\mathrm{b}} \times 10^{-7}$ & $\begin{array}{l}\text { Molar ratio, } \\
\text { Ag/AgSCN }^{c}\end{array}$ \\
\hline $\mathrm{M}_{1}$ & 2.233 & 18.987 & 1.325 & 3.177 & 0.024 \\
\hline $\mathrm{M}_{2}$ & 2.111 & 18.719 & 1.235 & 5.723 & 0.0463 \\
\hline $\mathrm{M}_{3}$ & 2.557 & 18.631 & 1.489 & 8.014 & 0.0538 \\
\hline $\mathrm{M}_{4}$ & 2.431 & 18.502 & 1.406 & 9.127 & 0.0649 \\
\hline $\mathrm{M}_{5}$ & 3.008 & 18.358 & 1.726 & 13.375 & 0.0775 \\
\hline
\end{tabular}

aMolar content of $\mathrm{AgSCN}$ is calculated as the mole of $\mathrm{AgSCN}=A \cdot B / 32$ (where $A$ is the atomic \% of $\mathrm{S}$ and $B$ is the weight (mg) of $\mathrm{Ag} @ \mathrm{AgSCN}$. ${ }^{\mathrm{b}} \mathrm{Molar}$ content of $\mathrm{Ag}$ is calculated according to $\mathrm{Ag}=(B-(A \cdot B / 32) \cdot 165.95) / 107.87$ (where $A$ is the atomic \% of $S$ and $B$ is the weight (mg) of $A g @ A g S C N$. $\mathrm{c}^{\mathrm{C}}$ Molar ratio of $\mathrm{Ag} / \mathrm{AgSCN}$ is calculated as $\mathrm{Ag} / \mathrm{AgSCN}=\mathrm{B} / \mathrm{A}$.
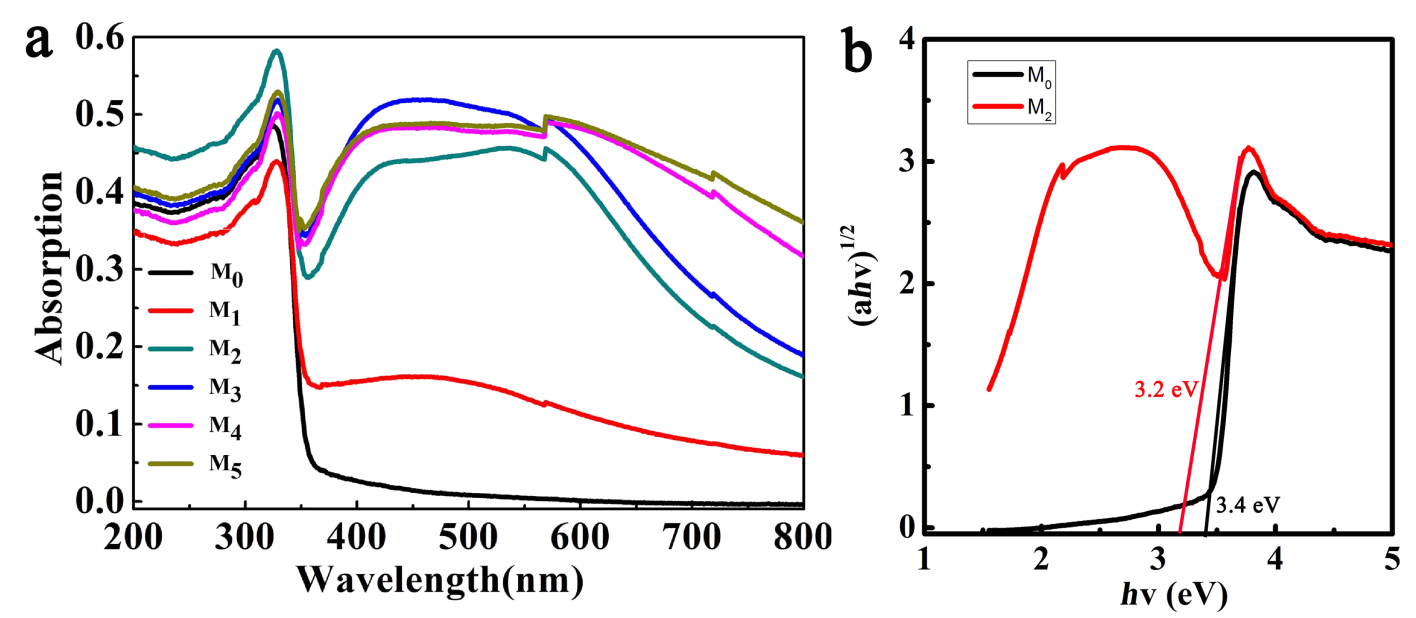

Figure 2: (a) UV-vis diffuse reflectance spectra of $M_{0}, M_{1}, M_{2}, M_{3}, M_{4}, M_{5}$. (b) Kubelka-Munk plots of $M_{0}$ and $M_{2}$. 
that can improve the visible-light catalytic activity of $\mathrm{AgSCN}$ $[31,32]$.

\section{Formation mechanism of Ag@AgSCN nanostructures}

To investigate the formation mechanism of $\mathrm{Ag} @ \mathrm{AgSCN}$ nanostructures, a time-dependent precipitation experiment was conducted. It can be seen that small AgSCN nanoplates with size of $\approx 10 \mathrm{~nm}$ were formed in $2 \mathrm{~min}$ due to the low solubility product constant $\left(K_{\mathrm{sp}}\right)$ of AgSCN (Figure 3a). These nanoplates rapidly aggregated due to the high surface energy, and the crystal growth occurred simultaneously (Figure $3 b$ ). At this time, the dropwise addition of the $\mathrm{AgNO}_{3}$ solution finished. The oriented-aggregates with a size of $\approx 1.5 \mu \mathrm{m}$ were formed in $10 \mathrm{~min}$ (Figure $3 \mathrm{c}$ ). Further extending the reaction time led to the gradual increase in the size of the nanostructures, and the sphere-like nanostructures with size of $\approx 2 \mu \mathrm{m}$ were finally obtained (Figure $3 \mathrm{~d}$ ). Certainly, because of the existence of $\mathrm{N}_{2} \mathrm{H}_{4} \cdot \mathrm{H}_{2} \mathrm{O}$, a small amount of Ag was formed at the same time, but it cannot be distinguished from the TEM image. So the XPS spectra of the samples obtained with and without $\mathrm{N}_{2} \mathrm{H}_{4} \cdot \mathrm{H}_{2} \mathrm{O}$ were compared (Figure 4a). As to the AgSCN nanostructures formed without $\mathrm{N}_{2} \mathrm{H}_{4} \cdot \mathrm{H}_{2} \mathrm{O}$, the bonding energy of $\mathrm{Ag} 3 \mathrm{~d}_{3 / 2}$ and Ag $3 \mathrm{~d}_{5 / 2}$ located at $373.35 \mathrm{eV}$ and $367.8 \mathrm{eV}$ with a symmetrical profile, indicated that there was no $\mathrm{Ag}^{0}$ in the sample. For $M_{1}$ (Figure $4 b$ ), the peaks of $\mathrm{Ag} 3 \mathrm{~d}_{5 / 2}$ and $\mathrm{Ag} 3 \mathrm{~d}_{3 / 2}$ can be divided into two different peaks at $368.2,368.0 \mathrm{eV}$ and 374.3 , $373.9 \mathrm{eV}$, respectively. The peaks at 368.2 and $374.3 \mathrm{eV}$ can be attributed to $\mathrm{Ag}^{0}$, indicating the presence of $\mathrm{Ag}^{0}$ [33]. The elemental analysis results shown in Table 1 also indicate the existence of a small amount of $\mathrm{Ag}^{0}$ in the precipitated products.

Further experiments indicated that $\mathrm{N}_{2} \mathrm{H}_{4} \cdot \mathrm{H}_{2} \mathrm{O}$ did not affect the morphology of the product. As shown in Figure S2a (see Sup-
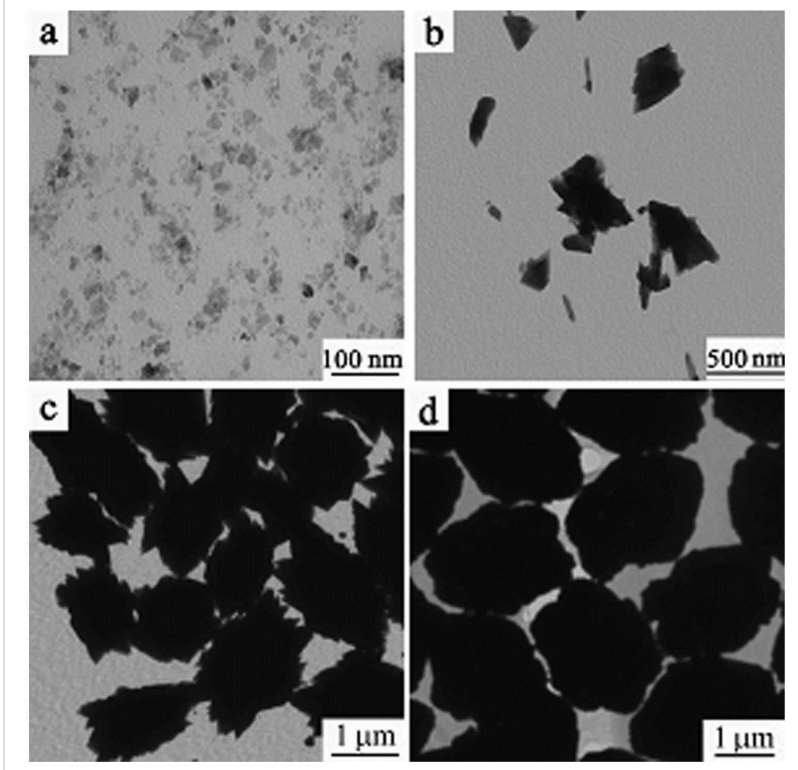

Figure 3: TEM images of precipitated samples formed after addition of the $\mathrm{AgNO}_{3}$ solution after (a) $2 \mathrm{~min}$, (b) $6 \mathrm{~min}$, (c) $10 \mathrm{~min}$, and (d) $20 \mathrm{~min}$.

porting Information File 1), the phase-pure AgSCN with the same morphology as the precipitated product was formed without the addition of $\mathrm{N}_{2} \mathrm{H}_{4} \cdot \mathrm{H}_{2} \mathrm{O}$ while other conditions were held constant, indicating the existence of $\mathrm{N}_{2} \mathrm{H}_{4} \cdot \mathrm{H}_{2} \mathrm{O}$ and the formation of the small amount of Ag had no effect on the morphology of the product. Figure S2b (see Supporting Information File 1) shows the SEM image of the sample obtained without polyvinylpyrrolidone (PVP). The product was composed of nanoparticles with of tens of nanometers in diameter with a large size distribution. This result revealed that the formation of the nanoplate units which composed the Ag@AgSCN nanostructures was directed by PVP. According to the previous
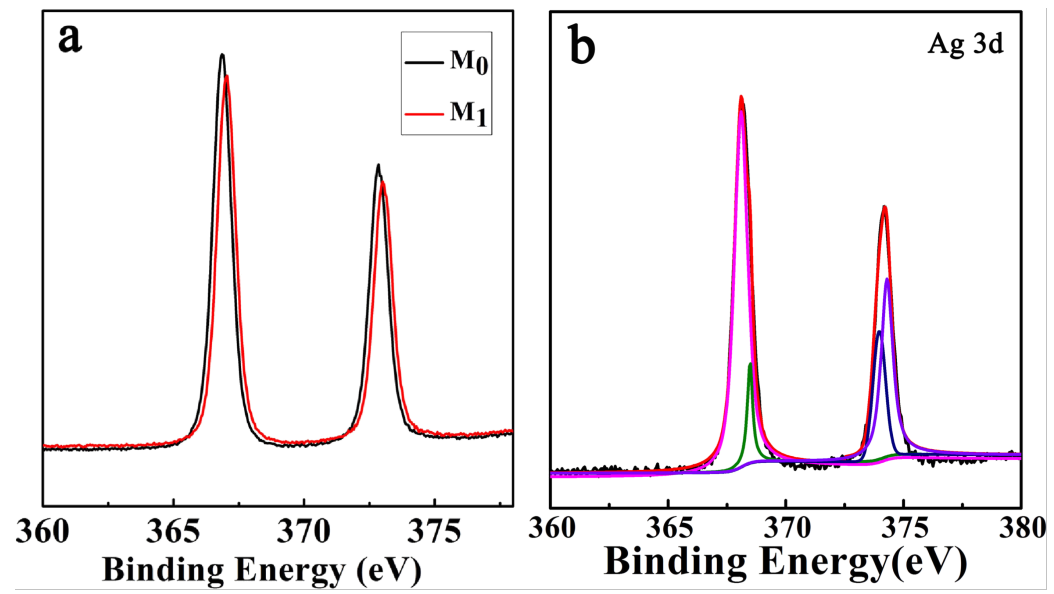

Figure 4: (a) XPS spectra of $M_{0}$ and $M_{1}$; (b) XPS peaks of $A g 3 d_{5 / 2}$ and $A g 3 d_{3 / 2}$ of $M_{1}$. 
studies, it was proposed that PVP can selectively adsorb on certain crystal planes and then lead to the formation of $\mathrm{AgSCN}$ nanoplates [34]. The rapid addition of the $\mathrm{AgNO}_{3}$ solution (instead of dropwise addition) resulted in the formation of crisscross nanostructures composed of nanoplates without small plates in the cross gap (see Supporting Information File 1, Figure S2c). BET measurements were used to illustrate the specific surface area of the nanostructured Ag@AgSCN, which was $4.04 \mathrm{~m}^{2} \cdot \mathrm{g}^{-1}$, while the BET specific surface area of a sample without PVP (see Supporting Information File 1, Figure $\mathrm{S} 2 \mathrm{~b})$ was only $2.88 \mathrm{~m}^{2} \cdot \mathrm{g}^{-1}$.

Based on the above results, the formation process of $\mathrm{Ag} @ \mathrm{AgSCN}$ nanostructures can be summarized. The AgSCN nanoplates were quickly formed under the direction of PVP once the $\mathrm{AgNO}_{3}$ solution was added into the solution (Figure 3a). Then rapid aggregation of the nanoplates occurred to reduce the total surface energy, with the simultaneously growth of the AgSCN plates (Figure 3b). The nanoplates were arranged in a face-to-face stacking manner, and the tetragonal star-shaped Ag@AgSCN nanostructures composed of nanoplates were formed. After that, the addition of the $\mathrm{AgNO}_{3}$ solution finished and the homogeneous nucleation stopped due to the decrease in concentration of the $\mathrm{Ag}(\mathrm{I})$ ions. Then, the heterogeneous nucleation on the nanoplate surface occurred and some small nanoplates gradually filled in the crisscross gap of the star-shaped Ag@AgSCN nanostructures. At last, the spherelike Ag@AgSCN nanostructures composed of nanoplates were formed.

In the following UV-light-induced reduction process, the content of Ag gradually increased with increased irradiation time. Due to the high stability, it is difficult for phase-pure $\mathrm{AgSCN}$ to be reduced to Ag by UV-light irradiation, but the existence of a small amount of $\mathrm{Ag}$ in the above precipitate can promote the transformation from AgSCN to Ag. Finally, the $\mathrm{Ag} @ \mathrm{AgSCN}$ nanostructures with different Ag content were obtained by adjusting the irradiation time of the UV light.

\section{Visible-light catalytic performance}

The visible-light catalytic activity of the samples was evaluated by measuring the degradation rate of oxytetracycline $\left(20 \mathrm{mg} \cdot \mathrm{L}^{-1}\right)$ under a halogen lamp $(400 \mathrm{~W})$ using phase-pure $\mathrm{AgSCN}$ as a reference. The oxytetracycline concentration variation with irradiation time for the $\mathrm{M}_{0}, \mathrm{M}_{1}, \mathrm{M}_{2}, \mathrm{M}_{3}, \mathrm{M}_{4}$ and $\mathrm{M}_{5}$ catalysts are shown in Figure 5a. The degradation rates were $29 \%, 81 \%, 84 \%, 61 \%, 49 \%$, and $30 \%$, respectively, in $60 \mathrm{~min}$ according to the absorption intensity at $364 \mathrm{~nm}$. When $\mathrm{M}_{2}$ was
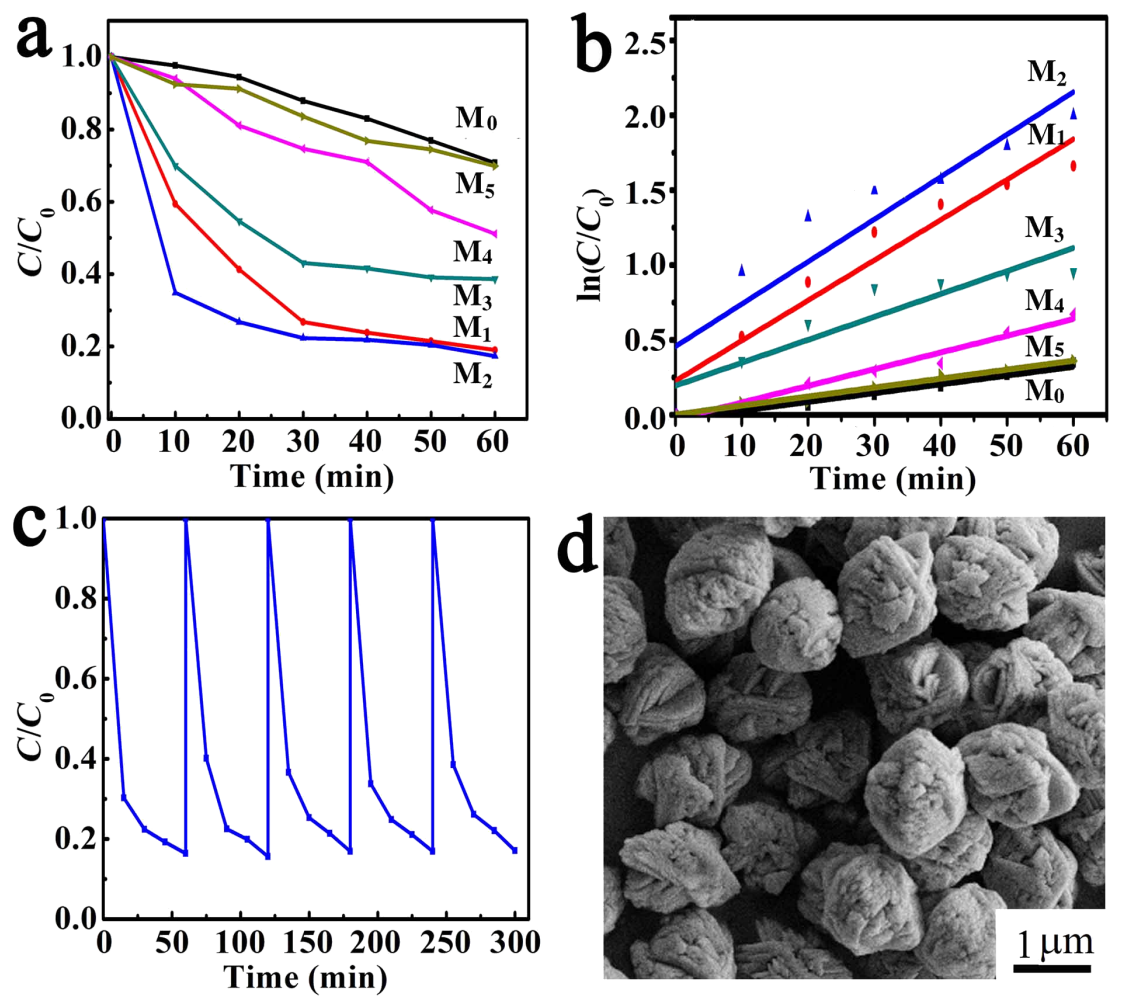

Figure 5: (a) Photocatalytic degradation of oxytetracycline over $M_{0}, M_{1}, M_{2}, M_{3}, M_{4}, M_{5}$. (b) Kinetic curves for the oxytetracycline degradation with $M_{0}, M_{1}, M_{2}, M_{3}, M_{4}, M_{5}$. (c) The cycle curves of oxytetracycline degradation using $M_{2}$ as a photocatalyst. (d) TEM images of $M_{2}$ after five cyclic experiments. 
used as a photocatalyst, the maximum degradation rate was obtained. The time-dependent UV-vis spectrum of the oxytetracycline solution over $\mathrm{M}_{2}$ is shown in Supporting Information File 1, Figure S3. Furthermore, the degradation efficiency of $\mathrm{Ag} @ \mathrm{AgSCN}$ was much higher than that of the pure AgSCN, mainly due to the localized surface plasmon resonance state of Ag particles in the visible-light region. The photogenerated electrons of AgSCN will be captured by Ag particles, rather than recombination with holes. The dipole character of Ag particles in the plasma can promote the separation of electrons and holes in AgSCN under visible light. The electrons will quickly transfer onto the surface of Ag nanoparticles, while the holes will congregate on the surface of AgSCN [35]. On one hand, with increasing Ag content in the product, the recombination of electrons and holes is inhibited, and the catalytic efficiency would be enhanced. But on the other hand, the formation of excess Ag on the surface of AgSCN particles would partially cover the active sites and reduce the adsorption of oxytetracycline on the AgSCN particle surface. Furthermore, the presence of excess Ag particles will decrease the absorption of light and reduce the production of electrons and holes. All of these phenomena led to the decrease of the degradation efficiency [36,37]. Therefore, with the increase of the Ag content in the $\mathrm{Ag} @ \mathrm{AgSCN}$ nanostructures, the degradation efficiency firstly increased and then decreased. The Langmuir-Hinshelwood model was used to investigate the kinetic behaviors of the photocatalyst for degradation of oxytetracycline [38]. Figure 5b showed that the photodegradation process of oxytetracycline nearly complied with the first-order kinetics, and the corresponding reaction rate constants $\left(K_{\mathrm{a}}\right)$ with $\mathrm{M}_{0}, \mathrm{M}_{1}, \mathrm{M}_{2}, \mathrm{M}_{3}, \mathrm{M}_{4}$ and $\mathrm{M}_{5}$ as catalysts were $0.0058,0.0269,0.02832,0.01531$, $0.01115,0.006 \mathrm{~min}^{-1}$, respectively. The value of $K_{\mathrm{a}}$ for $\mathrm{M}_{2}$ was 4.8-fold faster than that of the bare $\mathrm{AgSCN}$, so the existence of an appropriate amount of Ag played a prominent role in the photodegradation process. Electrochemical impedance spectrum (EIS) was used to illustrate the rate of charge transfer for the photocatalysts. Nyquist plots of $\mathrm{M}_{0}, \mathrm{M}_{1}, \mathrm{M}_{2}, \mathrm{M}_{3}, \mathrm{M}_{4}, \mathrm{M}_{5}$ were shown in Figure $\mathrm{S} 4$ (see Supporting Information File 1), the circular radius of plot over $\mathrm{M}_{2}$ in the high-frequency region was much smaller than that of other catalysts, which suggested the smallest charge transfer resistance for $\mathrm{M}_{2}$, while the charge transfer resistance for $\mathrm{M}_{0}$ was the largest. That can be attributed to the presence of Ag particles and the promotion of the separation of photogenerated carriers.

The stability of the plasmonic photocatalyst $\mathrm{M}_{2}$ was investigated by the degradation of oxytetracycline, which was of practical importance for application. As shown in Figure 5c, about $84 \%$ oxytetracycline was degraded in $60 \mathrm{~min}$ in the fifth cycle, and the efficiency was almost the same as for the first cycle. Further investigations revealed that after five cyclic experi- ments, the microstructure of $\mathrm{Ag} @ \mathrm{AgSCN}$ had no obvious changes except a somewhat smoother Ag@AgSCN nanostructure surface (Figure 5d). The composition of Ag, S, C, N in $\mathrm{M}_{2}$ hardly changed in the EDS analysis (see Supporting Information File 1, Figure S5), indicating that AgSCN cannot be reduced in this period of time under visible-light irradiation. Elemental analysis of the $\mathrm{Ag} @ \mathrm{AgSCN}$ after cyclic experiments were also performed. The result showed that the molar ratio of $\mathrm{Ag}$ to $\mathrm{AgSCN}$ is 0.047 , which was near the value in the fresh $\mathrm{M}_{2}$. All these results indicated that the prepared Ag@AgSCN nanostructures showed long-term stability as a photocatalyst. For Ag@AgSCN, Ag particles coated the surface of AgSCN uniformly, which trapped the photogenerated electrons and protected AgSCN from being reduced. Compared with the previously reported silver halogen photocatalysts $(\mathrm{Ag} / \mathrm{AgX}(\mathrm{X}=\mathrm{Cl}$, $\mathrm{Br}, \mathrm{I})$ ), the present $\mathrm{Ag} @ \mathrm{AgSCN}$ nanostructures exhibited superior catalytic stability $[13,14]$. This was mainly due to the higher stability of $\mathrm{AgSCN}$ than the $\mathrm{AgX}(\mathrm{X}=\mathrm{Cl}, \mathrm{Br}, \mathrm{I})$. Also, the stronger electron affinity of $\mathrm{SCN}^{0}$ as compared to $\mathrm{Br}^{0}$ and $\mathrm{Cl}^{0}$, and the more easy combination of $\mathrm{SCN}^{-}$with holes as compared to $\mathrm{Cl}^{-}$and $\mathrm{Br}^{-}$can improve the photocatalytic stability of Ag@AgSCN nanostructures.

Based on the above discussion, Ag nanoparticles located on AgSCN played a decisive role in the photocatalytic efficiency and stability of Ag@AgSCN. For one, Ag nanoparticles can absorb the visible light, which enhances the utilization of visible light compared with pure AgSCN. On the other hand, the presence of $\mathrm{Ag}$ particles effectively reduced the recombination of photogenerated electrons and holes, improving the efficiency and stability of the photocatalyst. The surface plasmon resonance of Ag nanoparticles occurred under visible light, and electrons in the metal from the Fermi level directly transferred to the conduction band of $\mathrm{AgSCN}$, whereas the vacancies were left on the surface of the Ag particles. The dipole-based resonance energy can directly excite semiconductors to produce photogenerated electron-hole pairs. The presence of Ag nanoparticles can not only improve the photocatalytic efficiency, but also can trap photogenerated electrons, slow the reduction of AgSCN, and enhance the stability of $\mathrm{Ag} @ \mathrm{AgSCN}$. The degradation mechanism suggested that electrons of $\mathrm{Ag} @ \mathrm{AgSCN}$ were prompted to the conduction band and holes were left in the valence band of AgSCN under irradiation (Figure 6a). The electrons were rapidly trapped by $\mathrm{Ag}$ nanoparticles, then captured by dissolved $\mathrm{O}_{2}$, resulting in the formation of $\bullet \mathrm{O}_{2}{ }^{-}$radicals, which can decompose oxytetracycline. The photogenerated holes can oxidize $\mathrm{H}_{2} \mathrm{O}$ into $\bullet \mathrm{OH}$, which could also oxidize oxytetracycline. To investigate the effect of $\cdot \mathrm{O}_{2}{ }^{-}$radicals, 1 , 4-benzoquinone (BQ), which was an efficient scavenger for - $\mathrm{O}_{2}{ }^{-}$[39], was added to the solution. The degradation efficiency of oxytetracycline was significantly suppressed as shown 

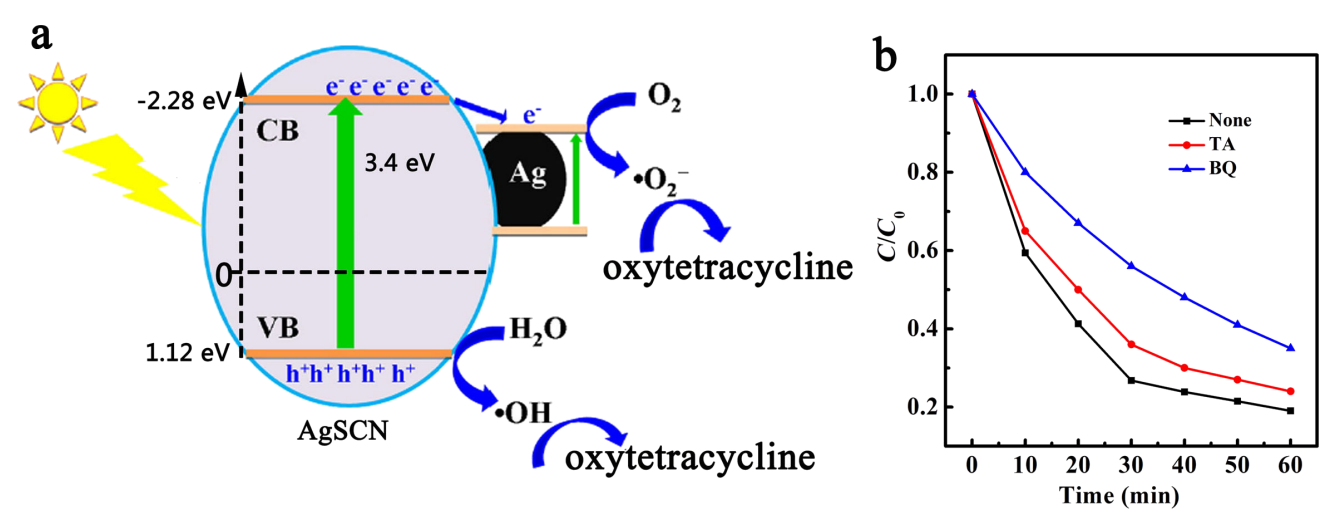

Figure 6: (a) Photocatalysis mechanism of the Ag@AgSCN plasmonic photocatalyst. (b) Photocatalytic degradation of oxytetracycline in the absence and presence of scavengers.

in Figure 6b. Additionally, the presence of $\bullet \mathrm{OH}$ was confirmed by addition of terephthalic acid (TA), which was a $\bullet \mathrm{OH}$ scavenger [40]. The degradation efficiency of oxytetracycline was also slowed, but was higher than that of the rate with $\mathrm{BQ}$. This revealed that the $\cdot \mathrm{O}_{2}{ }^{-}$and $\bullet \mathrm{OH}$ both contributed to the decomposition of oxytetracycline, but $\cdot \mathrm{O}_{2}{ }^{-}$was the main active species in the photocatalytic process.

\section{Conclusion}

$\mathrm{Ag} @ \mathrm{AgSCN}$ nanostructures composed of nanoplates were synthesized through a simple precipitation method, followed by UV-light irradiation. The sphere-like Ag@AgSCN nanostructures went through the formation of AgSCN nanoplates and their oriented aggregation, as well as simultaneous crystal growth. PVP played a critical role in the formation of 2D AgSCN units. The content of Ag in Ag@AgSCN nanostructures can be controlled by simply adjusting the irradiation time of the UV light. The catalytic performance of the nanostructure was evaluated by degradation of oxytetracycline under visiblelight irradiation. The Ag@AgSCN nanostructures showed high photocatalytic activity due to their surface plasmon resonance effects. When the molar ratio of $\mathrm{Ag} / \mathrm{AgSCN}$ was 0.0463 , the catalytic rate reached to the maximum and the value of $K_{\mathrm{a}}$ was 4.8-fold faster than that of the bare AgSCN. Most importantly, the prepared Ag@AgSCN nanostructures also exhibited superior photocatalytic stability compared to the previously reported silver halogen plasma catalyst, which make it more suitable for practical application.

\section{Experimental Chemicals}

Silver nitrate $\left(\mathrm{AgNO}_{3}\right.$, Shanghai Chemical Co. $)$ and ammonium thiocyanate $\left(\mathrm{NH}_{4} \mathrm{SCN}\right.$, Tianjin Reagent $\mathrm{Co}$.) were used as precursors for the synthesis of silver/silver thiocyanate nanostructures (Ag@AgSCN). Polyvinylpyrrolidone (PVP, K30,
Beijing Reagent Co. $)$ and hydrazine hydrate $\left(\mathrm{N}_{2} \mathrm{H}_{4} \cdot \mathrm{H}_{2} \mathrm{O}\right.$, 85 wt \%, Shanghai Chemical Co.) were used as stabilizer and reducing agent, respectively. For the photocatalytic test, oxytetracycline was purchased from Sinopharm Chemical Co. Ltd (China). All chemicals were used as received without further purification.

\section{Synthesis of $\mathrm{Ag} @ A g S C N$ nanostructures}

$\mathrm{Ag} @ \mathrm{AgSCN}$ nanostructures were prepared by photoreducing AgSCN nanoparticles. Due to the slow reducing rate of AgSCN under UV-vis light irradiation, the AgSCN nanoparticles (with a small amount of Ag nuclei) were firstly synthesized as follows. At first, $50 \mathrm{~mL}$ of deionized water, $5.55 \mathrm{~g}$ of PVP, $0.38 \mathrm{~g}$ of $\mathrm{NH}_{4} \mathrm{SCN}$ and $2 \mathrm{~mL} \mathrm{~N} \mathrm{H}_{4} \cdot \mathrm{H}_{2} \mathrm{O}$ (85 wt \%) were sequentially added to the beaker, and then $10 \mathrm{~mL}$ solution of $\mathrm{AgNO}_{3}(0.5 \mathrm{M})$ was injected drop by drop via a pipette. The addition of the $\mathrm{AgNO}_{3}$ solution lasted for $\approx 6 \mathrm{~min}$, after that the beaker was kept at $30^{\circ} \mathrm{C}$ for 30 min under stirring and $\mathrm{AgSCN}$ nanostructures with a small amount of $\mathrm{Ag}$ nuclei were obtained (labeled as $\mathrm{M}_{1}$ ). The precipitate was collected by centrifugation and washed with water several times and then dried at $60{ }^{\circ} \mathrm{C}$ for $6 \mathrm{~h}$ in a vacuum drying box.

Next, $0.1 \mathrm{~g}$ of the above-described nanostructures was dispersed in $50 \mathrm{~mL}$ deionized water by ultrasonication. To establish the adsorption-desorption equilibrium, the dispersion was stirred for 30 min under dark prior to the light irradiation. Then the production was performed under UV-light irradiation by using a halogen lamp (400 W). The reaction lasted for 1, 2, 2.5, and $3 \mathrm{~h}$ to adjust the ratio of $\mathrm{Ag}$ to $\mathrm{AgSCN}$ (which are respectively labeled as $\mathrm{M}_{2}, \mathrm{M}_{3}, \mathrm{M}_{4}, \mathrm{M}_{5}$ ). The product was collected by centrifugation at $4000 \mathrm{rpm}$ for $5 \mathrm{~min}$ and washed with water for three times. In this process, part of AgSCN was transferred to $\mathrm{Ag}$ by photoreduction with the color of the solid changing from milk white to pale red, red brown and black. The samples were 
dried at $60{ }^{\circ} \mathrm{C}$ for $6 \mathrm{~h}$ in a vacuum drying box. Then the $\mathrm{Ag} @ \mathrm{AgSCN}$ nanostructures with different molar ratio of $\mathrm{Ag}: \mathrm{AgSCN}$ were obtained.

As a comparison, the pure $\mathrm{AgSCN}$ nanomaterial (labeled as $\mathrm{M}_{0}$ ) was also prepared using the same procedure as preparing $\mathrm{AgSCN}$ with a small amount of Ag except the addition of $\mathrm{N}_{2} \mathrm{H}_{4} \cdot \mathrm{H}_{2} \mathrm{O}$.

\section{Characterization}

The morphology and microstructure of the samples were characterized using a field-emission scanning electron microscope (FE-SEM, JSM-6700F), a transmission electron microscope (TEM, JEM 100-CXII) with an accelerating voltage of $80 \mathrm{kV}$, and a high-resolution TEM (HRTEM, GEOL-2010) with an accelerating voltage of $200 \mathrm{kV}$. Also, powder X-ray diffraction (XRD) patterns were collected on an X-ray diffractometer (Rigaku D/Max 2200 PC) with a graphite monochromator and $\mathrm{Cu} \mathrm{K} \alpha$ radiation $(\lambda=0.15148 \mathrm{~nm})$, while the tube voltage and electric current were held at $40 \mathrm{kV}$ and $20 \mathrm{~mA}$. The composition of the samples was tested by Vario EL III organic elemental analyzer (Germany Elmentar Company). Ultraviolet-visible (UV-vis) diffuse reflectance spectra (DRS) were recorded on an Agilent Cary $100 \mathrm{UV}$-vis spectrophotometer coupled to an integrating sphere with $\mathrm{BaSO}_{4}$ as reference. X-ray photoelectron spectra (XPS) were recorded on a Perkin-Elmer PHI-5300 ESCA spectrometer with a pass energy of $35.75 \mathrm{eV}$ and an $\mathrm{Al} \mathrm{K} \alpha$ line excitation source. $\mathrm{N}_{2}$ adsorption-desorption isotherms were determined by using a Quadrasorb SI apparatus to obtain the Brunauer-Emmett-Teller (BET) surface area. Electrochemical impedance spectroscopy (EIS) was recorded on a CHI660A electrochemical workstation (CH Instrument Company, Shanghai, China).

\section{Photocatalytic performance test}

The photocatalytic performance of the $\mathrm{Ag} @ \mathrm{AgSCN}$ nanostructures was evaluated by degradation of oxytetracycline $(20 \mathrm{mg} / \mathrm{L}$ aqueous solution) at ambient temperature and pressure. To establish the adsorption-desorption equilibrium, the dispersion was stirred for 30 min under dark prior to the light irradiation. The degradation was performed under visible-light irradiation by using a halogen lamp $(400 \mathrm{~W})$ with a UV cutoff filter $(\lambda \geq 420 \mathrm{~nm})$. The halogen lamp spectrum is commonly used to simulate the sunlight spectrum, in which the visible light is about $60-80 \%$. An $\approx 3 \mathrm{~mL}$ aliquot was taken every $10 \mathrm{~min}$ and centrifuged to remove the dispersed photocatalyst, and the supernatant was transferred to a quartz cuvette to measure the UV-vis absorption spectrum. The concentration change of oxytetracycline was monitored according to its maximum absorbance at $364 \mathrm{~nm}\left(\lambda_{\max }\right)$ recorded by a Perkin-Elmer Lambda35 UV-vis spectrometer.

\section{Supporting Information}

\section{Supporting Information File 1}

Additional experimental data.

The Supporting Information contains details of the AgSCN characterization, Nyquist plots, EDS spectrum of different $\mathrm{Ag} / \mathrm{AgSCN}$ samples, and UV-vis absorption spectra of oxytetracycline solutions with $\mathrm{M}_{2}$ as a catalyst. [http://www.beilstein-journals.org/bjnano/content/ supplementary/2190-4286-8-277-S1.pdf]

\section{Acknowledgements}

This work is supported by the National Natural Science Foundation of China (Grant 21771118), and the Taishan Scholars Climbing Program of Shandong Province (tspd20150201).

\section{ORCID ${ }^{\circledR}$ iDs}

Dairong Chen - https://orcid.org/0000-0001-6961-9328

Xiuling Jiao - https://orcid.org/0000-0002-4358-7396

\section{References}

1. Kelsic, E. D.; Zhao, J.; Vetsigian, K.; Kishony, R. Nature 2015, 521, 516-519. doi:10.1038/nature14485

2. Cui, W.; Li, J.; Dong, F.; Sun, Y.; Jiang, G.; Cen, W.; Lee, S. C.; Wu, Z. Environ. Sci. Technol. 2017, 51, 10682-10690. doi:10.1021/acs.est.7b00974

3. Dong, F.; Xiong, T.; Sun, Y.; Lu, L.; Zhang, Y.; Zhang, H.; Huang, H.; Zhou, Y.; Wu, Z. Appl. Catal., B: Environ. 2017, 219, 450-458. doi:10.1016/j.apcatb.2017.07.082

4. Cui, W.; Li, J.; Cen, W.; Sun, Y.; Lee, S. C.; Dong, F. J. Catal. 2017, 352, 351-360. doi:10.1016/j.jcat.2017.05.017

5. Cavalcante, R. P.; Dantas, R. F.; Bayarri, B.; González, O.; Giménez, J.; Esplugas, S.; Junior, A. M. Catal. Today 2015, 252, 27-34. doi:10.1016/j.cattod.2014.09.030

6. Wang, Y.; Feng, C.; Zhang, M.; Yang, J.; Zhang, Z. Appl. Catal., B 2010, 100, 84-90. doi:10.1016/j.apcatb.2010.07.015

7. Meng, F.; Hong, Z.; Arndt, J.; Li, M.; Zhi, M.; Yang, F.; Wu, N. Nano Res. 2012, 5, 213-221. doi:10.1007/s12274-012-0201-x

8. Cao, Y.; Li, C.; Li, J.; Li, Q.; Yang, J. Nanoscale Res. Lett. 2015, 10, 251. doi:10.1186/s11671-015-0952-X

9. Kuai, L.; Geng, B.; Chen, X.; Zhao, Y.; Luo, Y. Langmuir 2010, 26, 18723-18727. doi:10.1021/la104022g

10. Hou, W.; Cronin, S. B. Adv. Funct. Mater. 2013, 23, 1612-1619. doi:10.1002/adfm.201202148

11. Wang, P.; Huang, B.; Qin, X.; Zhang, X.; Dai, Y.; Wei, J.; Whangbo, M.-H. Angew. Chem., Int. Ed. 2008, 47, 7931-7933. doi:10.1002/anie.200802483

12. Chen, Y.-S.; Choi, H.; Kamat, P. V. J. Am. Chem. Soc. 2013, 135, 8822-8825. doi:10.1021/ja403807f

13. Tang, Y.; Jiang, Z.; Xing, G.; Li, A.; Kanhere, P. D.; Zhang, Y.; Sum, T. C.; Li, S.; Chen, X.; Dong, Z.; Chen, Z. Adv. Funct. Mater. 2013, 23, 2932-2940. doi:10.1002/adfm.201203379

14. Ma, B.; Guo, J.; Dai, W.-L.; Fan, K. Appl. Catal., B: Environ. 2013, 130-131, 257-263. doi:10.1016/j.apcatb.2012.10.026 
15. Wang, P.; Huang, B.; Qin, X.; Zhang, X.; Dai, Y.; Wei, J.; Whangbo, M.-H. Angew. Chem. 2008, 120, 8049-8051. doi:10.1002/ange.200802483

16. Wang, H.; Yang, X.; Zi, J.; Zhou, M.; Ye, Z.; Li, J.; Guan, Q.; Lv, P.; Huo, P.; Yan, Y. J. Ind. Eng. Chem. 2016, 35, 83-92. doi:10.1016/j.jiec.2015.12.023

17. Wang, P.; Huang, B.; Zhang, X.; Qin, X.; Jin, H.; Dai, Y.; Wang, Z.; Wei, J.; Zhan, J.; Wang, S.; Wang, J.; Whangbo, M.-H. Chem. - Eur. J. 2009, 15, 1821-1824. doi:10.1002/chem.200802327

18. Hu, C.; Peng, T.; Hu, X.; Nie, Y.; Zhou, X.; Qu, J.; He, H. J. Am. Chem. Soc. 2010, 132, 857-862. doi:10.1021/ja907792d

19. Lou, Z.; Huang, B.; Wang, P.; Wang, Z.; Qin, X.; Zhang, X.; Cheng, H.; Zheng, Z.; Dai, Y. Dalton Trans. 2011, 40, 4104-4110. doi:10.1039/c0dt01795g

20. Xia, Y.; Xiong, Y.; Lim, B.; Skrabalak, S. E. Angew. Chem., Int. Ed. 2008, 48, 60-103. doi:10.1002/anie.200802248

21. An, C.; Peng, S.; Sun, Y. Adv. Mater. 2010, 22, 2570-2574. doi:10.1002/adma.200904116

22. Chen, H.; Xiao, L.; Huang, J. Mater. Res. Bull. 2014, 57, 35-40. doi:10.1016/j.materresbull.2014.05.032

23. Zhang, S.; Zhang, S.; Song, L.; Wu, X.; Fang, S. Chem. Eng. J. 2014, 243, 24-30. doi:10.1016/j.cej.2014.01.015

24. Hu, J.-W.; Zhang, X. Powder Technol. 2014, 267, 240-244. doi:10.1016/j.powtec.2014.07.035

25. Lin, J.-D.; Li, Z.-H.; Li, J.-R.; Du, S.-W. Polyhedron 2007, 26, 107-114. doi:10.1016/j.poly.2006.07.034

26. Yang, M.; Ma, J. Appl. Surf. Sci. 2009, 255, 9323-9326. doi:10.1016/j.apsusc.2009.07.028

27. Zurmühl, C.; Wolf, S.; Feldmann, C. Z. Anorg. Allg. Chem. 2015, 641, 1510-1514. doi:10.1002/zaac.201500107

28. Soofivand, F.; Salavati-Niasari, M.; Mohandes, F. J. Ind. Eng. Chem. 2014, 20, 3780-3788. doi:10.1016/j.jiec.2013.12.079

29. Amornpitoksuk, P.; Suwanboon, S. Adv. Powder Technol. 2014, 25 , 1026-1030. doi:10.1016/j.apt.2014.02.001

30. Gong, J.; Cao, J.; Zhang, J.; Liao, Q.; Zhang, G.; Wu, X. Mater. Technol. (Abingdon, U. K.) 2017. doi:10.1080/10667857.2017.1336597

31. Wang, P.; Huang, B.; Dai, Y.; Whangbo, M.-H. Phys. Chem. Chem. Phys. 2012, 14, 9813-9825. doi:10.1039/c2cp40823f

32. Cushing, S. K.; Li, J.; Meng, F.; Senty, T. R.; Suri, S.; Zhi, M.; Li, M.; Bristow, A. D.; Wu, N. J. Am. Chem. Soc. 2012, 134, 15033-15041. doi:10.1021/ja305603t

33. Liu, Y.; Fang, L.; Lu, H.; Li, Y.; Hu, C.; Yu, H. Appl. Catal., B: Environ. 2012, 115-116, 245-252. doi:10.1016/j.apcatb.2011.12.038

34. Zeng, J.; Xia, X.; Rycenga, M.; Henneghan, P.; Li, Q.; Xia, Y. Angew. Chem. 2011, 50, 244-249. doi:10.1002/anie.201005549

35. Wang, P.; Huang, B.; Lou, Z.; Zhang, X.; Qin, X.; Dai, Y.; Zheng, Z.; Wang, X. Chem. - Eur. J. 2010, 16, 538-544. doi:10.1002/chem.200901954

36. Xiang, Q.; Meng, G. F.; Zhao, H. B.; Zhang, Y.; Li, H.; Ma, W. J.; Xu, J. Q. J. Phys. Chem. C 2010, 114, 2049-2055. doi:10.1021/jp909742d

37. Gyawali, G.; Adhikari, R.; Joshi, B.; Kim, T. H.; Rodríguez-Gonzalez, V.; Lee, S. W. J. Hazard. Mater. 2013, 263 , 45-51. doi:10.1016/j.jhazmat.2013.03.065

38. Niu, J.; Ding, S.; Zhang, L.; Zhao, J.; Feng, C. Chemosphere 2013, 93, 1-8. doi:10.1016/j.chemosphere.2013.04.043

39. Stylidi, M.; Kondarides, D. I.; Verykios, X. E. Appl. Catal., B: Environ. 2004, 47, 189-201. doi:10.1016/j.apcatb.2003.09.014
40. Choudhury, B.; Borah, B.; Choudhury, A. Photochem. Photobiol. 2012, 88, 257-264. doi:10.1111/j.1751-1097.2011.01064.x

\section{License and Terms}

This is an Open Access article under the terms of the Creative Commons Attribution License

(http://creativecommons.org/licenses/by/4.0), which permits unrestricted use, distribution, and reproduction in any medium, provided the original work is properly cited.

The license is subject to the Beilstein Journal of Nanotechnology terms and conditions:

(http://www.beilstein-journals.org/bjnano)

The definitive version of this article is the electronic one which can be found at:

doi:10.3762/bjnano.8.277 